Western University

Scholarship@Western

8-1-2010

\title{
Hyperpolarized (3)He magnetic resonance imaging-derived pulmonary pressure-volume curves
}

Stephen Choy

Andrew Wheatley

David G McCormack

Grace Parraga

Follow this and additional works at: https://ir.lib.uwo.ca/biophysicspub

Part of the Medical Biophysics Commons

\section{Citation of this paper:}

Choy, Stephen; Wheatley, Andrew; McCormack, David G; and Parraga, Grace, "Hyperpolarized (3)He magnetic resonance imaging-derived pulmonary pressure-volume curves" (2010). Medical Biophysics Publications. 117.

https://ir.lib.uwo.ca/biophysicspub/117 


\title{
Hyperpolarized ${ }^{3} \mathrm{He}$ magnetic resonance imaging-derived pulmonary pressure-volume curves
}

\author{
Stephen Choy, ${ }^{1}$ Andrew Wheatley, ${ }^{1}$ David G. McCormack, ${ }^{1,2}$ and Grace Parraga ${ }^{1,3,4}$ \\ ${ }^{1}$ Imaging Research Laboratories, Robarts Research Institute, and ${ }^{2}$ Division of Respirology, Department of Medicine, \\ ${ }^{3}$ Department of Medical Biophysics, and ${ }^{4}$ Graduate Program in Biomedical Engineering, The University of Western Ontario, \\ London, Ontario, Canada
}

Submitted 23 September 2009; accepted in final form 3 June 2010

\begin{abstract}
Choy S, Wheatley A, McCormack DG, Parraga G. Hyperpolarized ${ }^{3} \mathrm{He}$ magnetic resonance imaging-derived pulmonary pressurevolume curves. J Appl Physiol 109: 574-585, 2010. First published June 10, 2010; doi:10.1152/japplphysiol.01085.2009.—We aimed to evaluate the potential for the use of hyperpolarized helium-3 magnetic resonance imaging (MRI) apparent diffusion coefficient (ADC) surrogates of alveolar size, together with literature-based morphological parameters in a theoretical model of lung mechanics to simulate noninvasive transpulmonary pressure-volume curves. Fourteen exsmokers with chronic obstructive pulmonary disease (COPD) $(n=8$ stage II, $n=6$ stage III/IV COPD) and five age-matched neversmokers, provided written, informed consent and were evaluated at baseline and $26 \pm 2$ mo later ( $n=15$ subjects) using plethysmography, spirometry, and ${ }^{3} \mathrm{He}$ MRI at 3.0 T. Total lung capacity, residual volume, and literature-based morphological parameters were used with alveolar volumes derived from ${ }^{3} \mathrm{He}$ ADC to simulate noninvasive pressure-volume curves. The resultant anterior-posterior transpulmonary pressure gradient was significantly decreased for stage II COPD $(P<0.01)$ and stage III COPD subjects $(P<0.001)$ compared with healthy volunteers. Both COPD subgroups showed increased alveolar radius compared with healthy subjects $(P<0.01$, stage II COPD; $P<$ 0.001 , stage III COPD). In addition, surface area and surface tension were significantly increased in stage III COPD compared with healthy volunteers $(P<0.01)$. These results suggest that ${ }^{3} \mathrm{He}$ MRI provides a potential noninvasive approach to evaluate lung mechanics regionally and further supports the use of ADC values as a regional noninvasive probe of pulmonary microstructure and compliance.
\end{abstract}

helium-3 magnetic resonance imaging; apparent diffusion coefficient; mathematical modeling

NONINVASIVE IMAGING METHODS provide a way to detect and quantify pulmonary changes associated with chronic obstructive pulmonary disease (COPD) $(5,24,27,28,37,40)$. While $\mathrm{X}$-ray computed tomography (CT) has been the imaging tool of choice for clinical care and research studies over the last 3 decades (5), hyperpolarized helium-3 $\left({ }^{3} \mathrm{He}\right)$ magnetic resonance imaging (MRI) offers both complementary and unique anatomic and quantitative functional information based on the ventilation distribution in the lung $(4,7,23,46)$ and the measurement of structure at the alveolar level $(1,3,7,48)$.

Notably, and unique among COPD imaging methods, ${ }^{3} \mathrm{He}$ MRI can be used to provide quantitative information about the relative sizes of alveoli, acinar ducts, and airways in each volume element (voxel) through the ${ }^{3} \mathrm{He}$ apparent diffusion coefficient (ADC), which is related to the restriction of ${ }^{3} \mathrm{He}$ self-diffusion $(36,48)$. The ${ }^{3} \mathrm{He}$ ADC provides a surrogate

Address for reprint requests and other correspondence: G. Parraga, Imaging Research Laboratories, Robarts Research Institute, PO Box 5015, 100 Perth Dr., London, Ontario, Canada N6A 5K8 (e-mail: gep@imaging.robarts.ca). measurement of air space size because of a number of key characteristics of ${ }^{3} \mathrm{He}$ gas. In particular, ${ }^{3} \mathrm{He}$ is small with a corresponding high self-diffusion rate $(\mathrm{mm} / \mathrm{ms})$, it is chemically inert, and cannot be transported across intact biological tissues and membranes. Accordingly, ${ }^{3} \mathrm{He}$ magnetic resonance (MR) methods can be used that are sensitive to ${ }^{3} \mathrm{He}$ gas self-diffusion and provide a specific measure of the ${ }^{3} \mathrm{He}$ signal $(36,48)$ to generate the ADC. For diffusion times on the order of 2-3 ms, the average displacement of helium atoms by virtue of its self-diffusion is the same order of magnitude as alveolar diameters (a few hundred micrometers), providing a way to measure alveolar sizes wherever ${ }^{3} \mathrm{He}$ gas enters on inhalation (36). Values for ${ }^{3} \mathrm{He}$ ADC at $3.0 \mathrm{~T}$ and using short diffusion times (1-3 ms) range from $0.88 \mathrm{~cm}^{2} / \mathrm{s}$ for unrestricted free space (akin to an infinitely large container) to $0.66 \mathrm{~cm}^{2} / \mathrm{s}$ for an elderly COPD patient with forced expiratory volume in $1 \mathrm{~s}$ $\left(\mathrm{FEV}_{1}\right) 26 \%$ predicted (32) and $0.16 \mathrm{~cm}^{2} / \mathrm{s}$ for a young nonsmoker with $\mathrm{FEV}_{1} 130 \%$ predicted (33). Previous studies have shown that ADC correlates with pulmonary function (26) and histological measurements of lung surface area $(41,47)$ in COPD. Importantly, the ADC value itself has been found to be dependent on age (13), position (15,45), gravity (10), and smoking history (14), and is highly reproducible over short periods of time $(29,32)$. Recent studies have also shown that the distribution of $\mathrm{ADC}$ is dependent on direction $(15,40)$, and this dependency is different in diseased individuals, suggesting that ADC is sensitive to both anatomic location and disease.

A proposed mechanism for the anatomic distribution of the ${ }^{3} \mathrm{He}$ MRI ADC is the effect of the gravitational gradient on transpulmonary pressure (Ptp) $(14,15,40)$. Because our laboratory previously observed significant differences in ADC anatomic distribution between healthy subjects and stage III COPD (10) and because it has been well-established that Ptp is nonuniform and related to lung expansion and regional ventilation (25), we explored the utility of ADC values to provide regional alveolar volumes and generate regional Ptp-volume (PV) curves. Previously, Coxson and coworkers (6) reported $\mathrm{X}$-ray CT estimates of total and regional lung volumes and showed that pleural pressure gradients could be determined from the volume of gas per gram of lung tissue estimated from X-ray attenuation values in CT scans. CT measurements of the volume of gas per gram of tissue reflect the degree of emphysematous damage and gas trapping and are conceptually similar to the finding of regional differences in ADC measurements from ${ }^{3} \mathrm{He}$ MRI $(10,40)$. For example, the finding of relatively increased ADC in the posterior slices (thus increased alveolar size in the dependent portion of the subject) in COPD compared with age-matched healthy volunteers might be reflective of increased gas trapping in these individuals. 
Here we report the use of experimental ADC data as surrogate measurements of alveolar size in well-established lung micromechanical models $(2,20,38,42-44)$, reviewed in Ref. 21, to simulate Ptp gradients in healthy volunteers and COPD ex-smokers. The lung micromechanical models used morphological parameters, such as alveolar expansion, lung volume, lung compliance, and surface tension. Preliminary results from this pilot study provide further evidence $(41,47)$ of the potential for noninvasive lung imaging measurements of regional lung physiology.

\section{METHODS}

Study subjects. Nineteen subjects were evaluated and enrolled, as previously described (11), from the general population of the tertiary health care center, as well as directly from the COPD clinics at the three local teaching hospitals. All subjects provided written, informed consent to the study protocol, approved by the local research ethics board and Health Canada, and the study was compliant with the Personal Information Protection and Electronic Documents Act (Canada). COPD subjects required a disease diagnosis of at least $1 \mathrm{yr}$, having had a smoking history of at least 10 pack $\cdot y r$. Age-matched healthy subjects were included, if they had no history of chronic respiratory disease, $<1$ pack $\cdot y r$ smoking history, $\mathrm{FEV}_{1}>80 \%$ predicted, $\mathrm{FEV}_{1}$ divided by the forced vital capacity (FVC) or $\mathrm{FEV}_{1} / \mathrm{FVC}>70 \%$, and no current diagnosis or history of cardiovascular disease. Throughout the duration of the study, COPD subjects were to be withdrawn from the study, if they had experienced a COPD exacerbation, or if they experienced a drop in arterial oxygen levels as monitored using pulse oximetry below $80 \%$ for 15 continuous seconds during MRI procedures. However, no subjects were withdrawn for these reasons. COPD and healthy subjects were categorized according to Global Initiative for Chronic Obstructive Lung Disease (GOLD) criteria (35).

Study assessments. After subjects provided written, informed consent, they were screened for MRI and coil compatibility and underwent a physical exam, plethysmography, and spirometry. Spirometry and plethysmography were performed in the morning after patients delayed inhaled bronchodilators and corticosteroids for $\sim 12 \mathrm{~h}$, as previously described (34). Briefly, spirometry was performed at the MRI visit using an ndd EasyOne spirometer (ndd Medizintchnik AG, Zurich, Switzerland), reporting $\mathrm{FEV}_{1}$ (absolute and percent predicted) and FVC. COPD subjects performed spirometry at a pre-MRI screening visit (pre- and postbronchodilator) and were enrolled based on the postbronchodilator $\mathrm{FEV}_{1}$ measurement that was, furthermore, required to be within $3 \%$ of prebronchodilator $\mathrm{FEV}_{1}$, eliminating the potential for underlying asthma to confound results. Whole body plethysmography (MedGraphics, St. Paul, MN) was performed immediately before MRI for the measurement of total lung capacity (TLC), inspiratory capacity (IC), residual volume (RV), and functional residual capacity (FRC).

Hyperpolarized ${ }^{3} \mathrm{He}$ administration. For each subject, vital signs were measured, and arterial $\mathrm{O}_{2}$ levels were recorded before pre-MRI in the supine position to ensure the subject could tolerate MR scanning in a supine position. Spirometry was performed, as per American Thoracic Society standard, with the subject seated upright. Subjects were administered a practice dose of mixed ${ }^{4} \mathrm{He}$-nitrogen while seated outside the scanner. Digital pulse oximetry was used to monitor arterial blood oxygenation levels during MR scanning and all breath-hold maneuvers. Hyperpolarized ${ }^{3} \mathrm{He}$ gas was provided by a turnkey, spin-exchange polarizer system (HeliSpin, GEHC, Durham, $\mathrm{NC})$. In a typical study, this system provided $30 \%$ polarization in 12 h. Doses $(5 \mathrm{ml} / \mathrm{kg})$ were delivered in 1-liter plastic bags (Tedlar, Jensen Inert Products, Coral Springs, FL), diluted with ultrahigh purity, medical grade nitrogen (Spectra Gases, Alpha, NJ). Polarization of the diluted dose was quantified by a polarimetry station (GEHC, Durham, NC). ${ }^{3} \mathrm{He}$ MR scans were acquired during an inhalation breath hold after inspiration from $\mathrm{RV}$ of a 1-liter $5 \mathrm{ml} / \mathrm{kg}$ dose of ${ }^{3} \mathrm{He}$ mixed with $\mathrm{N}_{2}$. Post-MRI spirometry was also performed for all subjects.

Imaging. MRI was performed on a whole body 3.0-T Excite 12.0 MRI system (GEHC, Milwaukee, WI) with broadband imaging capability, as previously described (34). All helium imaging employed a whole body gradient set with maximum gradient amplitude of 1.94 $\mathrm{G} / \mathrm{cm}$ and a single-channel, rigid elliptical transmit/receive chest coil (RAPID Biomedical, Wuerzburg Germany). The basis frequency of the coil was $97.3 \mathrm{MHz}$, and excitation power was $3.2 \mathrm{~kW}$ using an AMT 3T90 RF power amplifier (GEHC, Milwaukee, WI).

A flip angle of $7^{\circ}$ was used, and ${ }^{3} \mathrm{He}$ multislice images were obtained in the coronal plane using a fast gradient-recalled echo method with centric $k$-space sampling (14). Two interleaved images (echo time $=3.7 \mathrm{~ms}$, repetition time $=7.6 \mathrm{~ms}, 128 \times 128,7$ slices, $30 \mathrm{~mm}$ thick, field of view $=40 \mathrm{~cm} \times 40 \mathrm{~cm}$ ), without and with additional diffusion sensitization (maximum gradient amplitude $\mathrm{G}=$ $1.94 \mathrm{G} / \mathrm{cm}$, rise and fall time $=0.5 \mathrm{~ms}$, duration $=0.46 \mathrm{~ms}, b$ value $=1.6 \mathrm{~s} / \mathrm{cm}^{2}$ ) were acquired for each slice. All scanning was completed within $7 \mathrm{~min}$ of the subject first lying in the scanner.

Image analysis. ADC images were analyzed by a single trained observer in an image visualization environment with room lighting levels equivalently established for all image analysis sessions. Mean $\mathrm{ADC}$ and $\mathrm{ADC}$ maps were processed using in-house software programmed in the IDL Virtual Machine platform [Research Systems, Denver, CO, as previously described (34)]. Briefly, the major airways were manually segmented from the images with both lungs remaining for analysis. The MR signal intensity acquired without diffusion sensitization $\left(S_{0}\right)$ and the MR signal intensity acquired with diffusion sensitization $(S)$ were used to calculate ADC, where $b=1.6 \mathrm{~s} / \mathrm{cm}^{2}$, as described in Eq. 1:

$$
\mathrm{ADC}=\frac{\ln \left(\frac{S_{0}}{S}\right)}{b}
$$

Five center slices or regions of interest (ROI) were examined, as shown schematically in Fig. 1, and the arithmetic mean and standard deviation (SD) of ADC were calculated from ADC histograms.

Theory for generation of alveolar volume. Acinar duct dimensions were generated from mean ADC values following a method described by Yablonskiy et al. (48). Briefly, the transverse attenuation exponent $\left(\Gamma_{\mathrm{T}}\right)$ was used as a function of the acinar duct radius $(R)$ and values of $\Gamma_{\mathrm{T}}$ were directly calculated using $E q .2$ :

$$
\Gamma_{\mathrm{T}}=\frac{4\left(\gamma \mathrm{G}_{\mathrm{m}} R^{2}\right)^{2} \delta}{D_{0}} \sum_{j} \frac{\beta_{1 j}^{-4}}{\left(\beta_{1 j}^{2}-1\right)} Q\left(\frac{D_{0} \delta}{R^{2}} \beta_{1 j}^{2}, \frac{\Delta}{\delta}, \frac{\tau}{\delta}\right)
$$

The function $Q$ was calculated as provided in equation 3 as:

$$
\begin{aligned}
& Q(\alpha, \eta, \varepsilon)=1- \frac{4}{3} \varepsilon-\frac{2}{\alpha^{3} \varepsilon^{2}}[\exp (-\alpha \varepsilon)+\alpha \varepsilon-1] \\
&+\frac{4}{\alpha^{3} \varepsilon^{2}} \sinh ^{2}\left(\frac{\alpha \varepsilon}{2}\right)\{\exp [-\alpha(1-\varepsilon] \\
&\left.\quad-2 \sinh ^{2}\left[\frac{\alpha(1-\varepsilon)}{2}\right] \exp (-\alpha \eta)\right\}
\end{aligned}
$$

where $\alpha=D_{0} \beta_{i j}^{2} \delta / R^{2}, \eta=\Delta / \delta$, and $\varepsilon=\tau / \delta, \gamma=2.04 \times 10^{8} \mathrm{~s}^{-1}, \mathrm{~T}^{-1}$ (gyromagnetic ratio), $\mathrm{G}_{\mathrm{m}}=1.95 \times 10^{-4} \mathrm{~T} / \mathrm{s}$ (maximum gradient amplitude), $D_{0}=0.88 \mathrm{~cm}^{2} / \mathrm{s}$ (free diffusion constant of ${ }^{3} \mathrm{He}$ ), $\beta_{1 j}$ is the $j$ th root of the first-order Bessel function, $\delta=1.46 \mathrm{~ms}$ (gradient pulse duration), $\Delta=2.94 \mathrm{~ms}$ (diffusion time), and $\tau=0.50 \mathrm{~ms}$ (ramp time).

We used a similar assumption and approximated equation (2) using the first term in the sum, because the ratio $R / l_{0}$ was $<2$. The $R$ was previously reported by Haefeli-Bleuer and Weibel (18) to be $\sim 350$ 


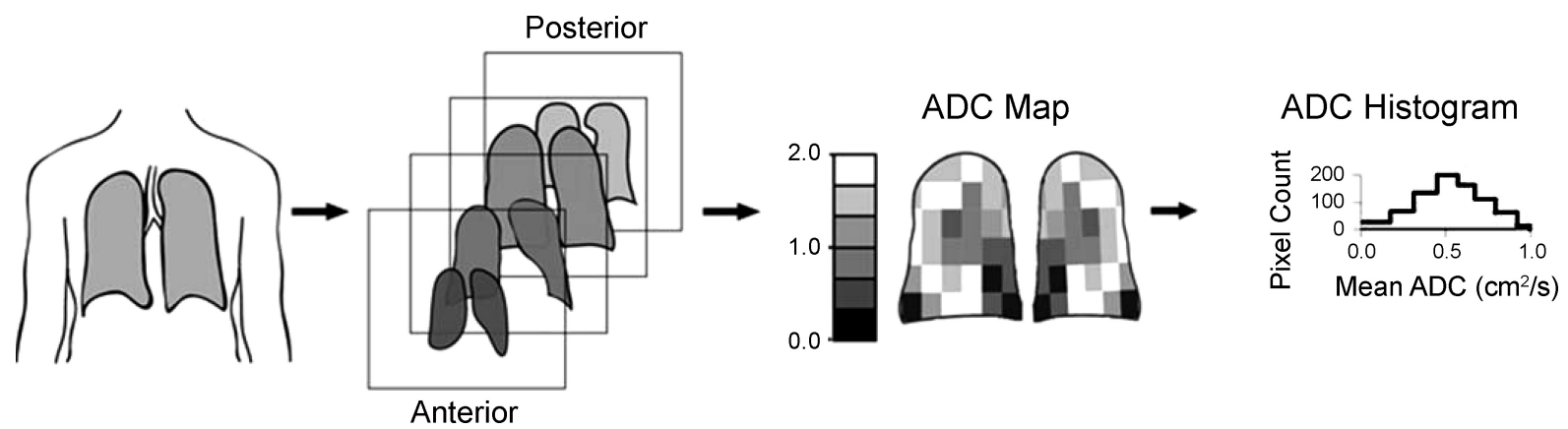

Fig. 1. Helium-3 $\left({ }^{3} \mathrm{He}\right)$ magnetic resonance imaging (MRI) for calculation of mean apparent diffusion coefficient (ADC) for regions of interest. ${ }^{3} \mathrm{He}$ diffusion-weighted MRI was acquired on a slice-by-slice basis. ADC maps were generated from the five center slices by computing ADC on a pixel-by-pixel basis. ADC histograms ( $y=$ frequency, $x=$ ADC value) were generated for each slice to derive mean ADC and standard deviation.

$\mu \mathrm{m}$, and the characteristic diffusion length $l_{0}$ for helium atoms was $720 \mu \mathrm{m}$ for the diffusion time used in this study (2.94 ms).

Additionally, the transverse attenuation exponent was calculated in relationship to the transverse diffusion coefficient $\left(D_{\mathrm{T}}\right)$ described in Eq. 4 by:

$$
D_{\mathrm{T}}=\frac{\Gamma_{\mathrm{T}}}{b}
$$

Yablonskiy related the $D_{\mathrm{T}}$ and longitudinal diffusion coefficient $\left(D_{\mathrm{L}}\right)$ with mean diffusion coefficient $\left(D_{\mathrm{M}}\right)$ by considering the acinar duct as a cylindrical object consisting of a tube embedded in the alveolar sleeve (Fig. 2), according to Haefeli-Bleuer and Weibel (18). This geometry gave rise to the following relationship described by Eq. 5:

$$
D_{\mathrm{M}}=\frac{1}{3} D_{\mathrm{L}}+\frac{2}{3} D_{\mathrm{T}}
$$

For the purposes of this study, $D_{\mathrm{M}}=\mathrm{ADC}$, and ratio of $D_{\mathrm{L}}$ to $D_{\mathrm{T}}$ $\left(D_{\mathrm{L}} / D_{\mathrm{T}}\right)$ for healthy and emphysematous subjects was determined empirically to be 3 (48). Thus Eq. 5 takes the form described by Eq. 6 :

$$
D_{\mathrm{T}}=\frac{3}{5} \mathrm{ADC}
$$

and relating this to $E q .4$ gives rise to $E q$. 7:

$$
\Gamma_{\mathrm{T}}=\frac{3}{5} \mathrm{ADC} \cdot b
$$

To estimate $R$, values of $\Gamma_{\mathrm{T}}$ were calculated over a range of radii $(R)$ using Eq. 2. Mean ADC was used in Eq. 7, and $b$ was set to $1.6 \mathrm{~s} / \mathrm{cm}^{2}$, which sets the right side of $E q .7$ to a single number. The chosen $R$ produced a value of $\Gamma_{\mathrm{T}}$ that satisfied $E q$. 7. The alveolar radius $(L)$ was estimated to be one-third the $R$ of the acinar duct, based on published morphometry estimates reported by Ingenito et al. (21).

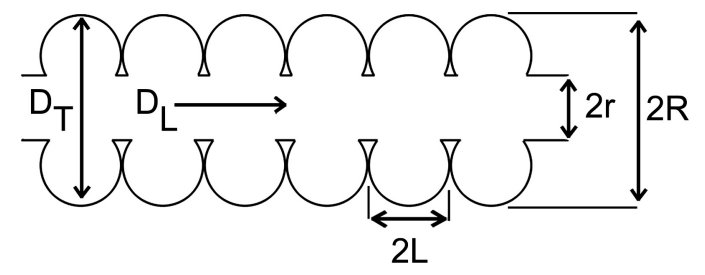

Fig. 2. Weibel acinar duct model. The schematic illustrates the structure of the acinar duct, considered as a cylinder with radius $(r)$ surrounded by numerous alveoli (open circles) that form an alveolar sleeve. The combined radius of the airway and alveolar sleeve is the acinar duct radius $(R)$, and the radius of the alveoli is $L$. The transverse $\left(D_{\mathrm{T}}\right)$ and longitudinal diffusion coefficient $\left(D_{\mathrm{L}}\right)$ are components of the mean ADC.
It is important to note the dependence of $E q .7$ on diffusion time as it assumes the diffusion length is on the order of the alveolar duct diameter. For diffusion time slightly larger or shorter than the one presented, one would expect a decrease in ADC and a simultaneous decrease in $\Gamma_{\mathrm{T}}$, which is itself dependent on diffusion time. The net effect is that the resulting $R$ should remain similar. At extremely long or short diffusion time, different lung structures will be probed (17), which would likely bias the calculation of $R$. To the author's knowledge, there has been no analysis on an optimal diffusion time for probing the $R$. For our diffusion time of $2.94 \mathrm{~ms}$, the diffusion length is $720 \mu \mathrm{m}$, which is very similar to the diameter of a human acinar duct (18).

Theory for generation of PV curves. PV curves were simulated using the micromechanical model derived by Stamenovic (38) and validated by Ingenito et al. (21) using published morphometry and biomechanical data. Ptp was expressed as related to lung volume (V) by $E q .8$ as follows:

$$
\operatorname{Ptp}(\mathrm{V})=\frac{N \mathrm{~F}(L) L}{3 \mathrm{~V}}+\frac{2}{3} \frac{\gamma(S) S}{\mathrm{~V}}+\frac{n \mathrm{~F}(l) l}{3 \mathrm{~V}}
$$

The first term is the contribution of the peripheral tissue network, which extends from the pleura into the parenchyma. The network is modeled as $N$ distinct line elements of length $L$, each contributing a force $\mathrm{F}(L)$ upon stretching. According to Ingenito et al. (21), $N$ is equivalent to the number of alveoli, and $L$ is equivalent to the average $L$. The second term describes the contribution of surface tension in the alveoli. The entire surface area $S$ of the alveoli is covered with surfactant, which has a surface tension $\gamma(S)$ that varies with $S$. The relationship between $S$ and $\gamma(S)$ is shown in Table 1 and is adapted from Ingenito et al. (21). The third term describes the contribution of the alveolar duct network, which Stamenovic (38) approximated by a series of circular rings embedded in the alveolar duct wall. Similar to the first term, there are $n$ distinct rings of circumference $l$ that contribute a force $\mathrm{F}(l)$. The force-length relationship $\mathrm{F}(L)$ and $\mathrm{F}(l)$

Table 1. Relationship between surface area and surface tension

\begin{tabular}{cc}
\hline \hline$S, \mathrm{~cm}^{2}$ & $\gamma(S), \mathrm{dyn} / \mathrm{cm}$ \\
\hline 965,096 & 25.5 \\
848,229 & 22 \\
738,901 & 17.5 \\
637,114 & 14 \\
542,866 & 10.5 \\
456,158 & 7 \\
376,990 & 3.5 \\
305,362 & 0 \\
\hline
\end{tabular}

$S$, surface area; $\gamma(S)$, surface tension. 
Table 2. Study subject demographics

\begin{tabular}{lccc}
\hline \hline & Healthy Volunteers & Stage II COPD & Stage III COPD \\
\hline$n$ & 5 & 8 & 6 \\
Age (range), yr & $69 \pm 6(58-74)$ & $68 \pm 6(59-74)$ & $68 \pm 6(62-75)$ \\
Male sex, no. & 2 & 4 & 4 \\
Body mass index & & & \\
$\quad$ (range) & $25 \pm 2(24-29)$ & $29 \pm 4(26-38)$ & $26 \pm 5(19-34)$ \\
FEV,$\%$ & $110 \pm 23$ & $64 \pm 8^{\mathrm{a}}$ & $37 \pm 6^{\mathrm{b}, \mathrm{c}}$ \\
FEV $1 / \mathrm{FVC}, \%$ & $77 \pm 4$ & $55 \pm 11^{\mathrm{d}}$ & $33 \pm 8^{\mathrm{b}, \mathrm{e}}$ \\
IC, \% & $106 \pm 18$ & $97 \pm 18$ & $77 \pm 20$ \\
RV, \% & $95 \pm 15$ & $142 \pm 22$ & $234 \pm 111$ \\
FRC, \% & $101 \pm 12$ & $116 \pm 15$ & $189 \pm 79$ \\
TLC, \% & $105 \pm 12$ & $107 \pm 10$ & $137 \pm 52$ \\
\hline
\end{tabular}

Values are means $\pm \mathrm{SD} ; n$, no. of subjects. COPD, chronic obstructive pulmonary disease; $\mathrm{FEV}_{1}$, forced expiratory volume in $1 \mathrm{~s} ; \%$, percent predicted; FVC, forced vital capacity; IC, inspiratory capacity; RV, residual volume; FRC, functional residual capacity; TLC, total lung capacity. ${ }^{\mathrm{a}} P<0.001$ for stage II COPD compared with healthy volunteers. ${ }^{\mathrm{b}} P<0.001$ for stage III COPD compared with healthy volunteers. ${ }^{\mathrm{c}} P<0.01$ for stage III COPD compared with stage II COPD. ${ }^{\mathrm{d}} P<0.01$ for stage II COPD compared with healthy volunteers. ${ }^{\mathrm{e}} P<0.001$ for stage III COPD compared with stage II COPD.

were taken from the work of Fukaya et al. (16), experimentally derived from strips of parenchymal tissue, and are expressed explicitly by Ingenito (21). The number of alveolar ducts is estimated by Ingenito to be one log less than the number of alveoli $(N)$. The ducts themselves contain a number of force-bearing fibers, and each fiber is then modeled as a series of alveolar duct rings. Thus we approximate the total number of alveolar duct rings $(n)$ to be equal to the number of alveoli $(N)$.

Parameter values for the model were obtained that were representative of disease status. $L$ was previously estimated from ADC measurements, which were obtained from ${ }^{3} \mathrm{He}$ scans at a static volume of RV +1 liter. $N$ was computed at RV +1 liter, assuming the alveoli were spherical, and contributed to the entire lung volume. Subsequent values of $L$ at arbitrary volumes $\mathrm{V}$ were calculated given $N=$ constant. $n, S$, and $\gamma(S)$ were calculated following the relationships described by Ingenito: $n=N, S$ was calculated assuming each alveoli was spherical with radius $L$, and $\gamma(S)$ was calculated using experimental data from Ingenito (21). It was assumed that the relationship between $\gamma(S)$ and $S$ was not changed due to emphysematous damage. For the calculation of $\mathrm{F}(L)$ and $\mathrm{F}(l)$, we used the form described by Ingenito in Eq. 9.

$$
\mathrm{F}(L)=\left(\frac{L}{L_{0}}-1\right) \exp \left[4.5\left(\frac{L}{L_{0}}-1\right)\right]
$$

where $L_{0}$ is the alveolar radius, and $l_{0}$ is the duct ring circumference at RV.

Exponential analysis. PV curves can be described between TLC and $50 \%$ TLC by the exponential function $(9,21)$ provided in Eq. 10:

$$
\mathrm{V}(\mathrm{P})=\mathrm{V}_{\max }-\left(\mathrm{V}_{\max }-\mathrm{V}_{\min }\right) \exp (-k \mathrm{P})
$$

where $\mathrm{V}$ is volume, $\mathrm{P}$ is $\mathrm{Ptp}$, and $k$ is the shape factor, which was previously described as a volume-independent index of pulmonary elasticity (9). We fitted each curve to Eq. 10 using an iterative least

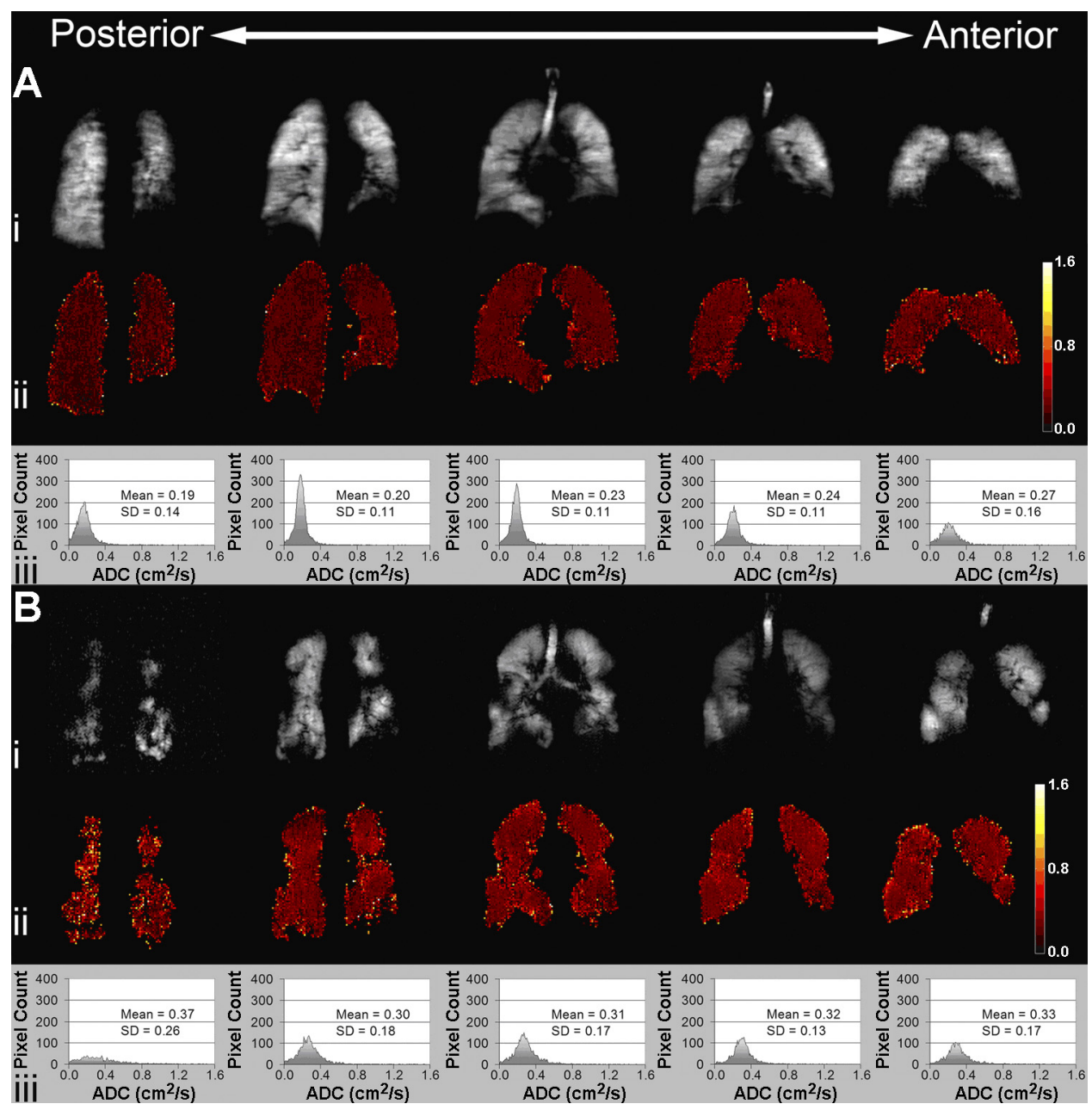

Fig. 3. Representative healthy subject's and stage III chronic obstructive pulmonary disease (COPD) subject's ${ }^{3} \mathrm{He}$ MRI ADC maps and histograms. Representative data are shown for one healthy elderly volunteer $(A)$ and one subject with stage III COPD $(B)$. For each subject, five ${ }^{3} \mathrm{He}$ diffusion-weighted MRI center slices (i) generated five ADC maps (ii) and ADC histograms (iii). 
Table 3. Subject listing of ADC and related parameters

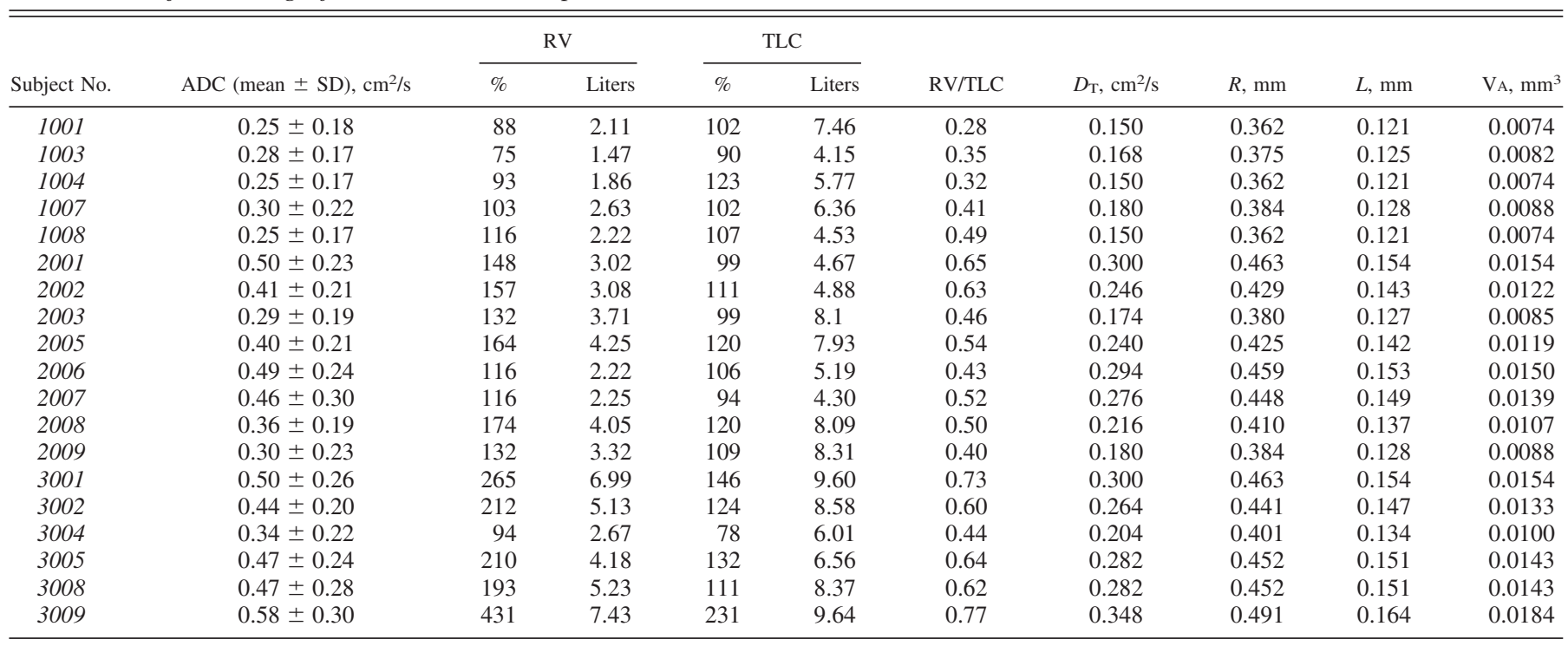

ADC, apparent diffusion coefficient; $D_{\mathrm{T}}$, transverse diffusion coefficients; $R$, acinar duct radii; $L$, alveolar radii; VA, alveolar volume. Healthy subjects, 1000 's; COPD stage II subjects, 2000's; COPD stage III subjects, 3000's.

squares method, and extracted the shape factor $k$ to compare our results across COPD cohorts and with the literature.

Statistics. Means for $\mathrm{ADC}$ and $\mathrm{ADC}_{\mathrm{SD}}$ were calculated as the arithmetic mean of the histogram describing each and every pixel value ADC vs. frequency. Comparison of subgroup demographics, which include age, body mass index, $\mathrm{FEV}_{1} \%$ predicted, $\mathrm{FEV}_{1} / \mathrm{FVC}$, IC \%predicted, RV \%predicted, FRC \%predicted, and TLC \%predicted, were performed using a one-way ANOVA with GraphPad Prism version 4.00 (GraphPad Software, San Diego, CA). Paired $t$-tests were used to compare the mean $k$ values of the simulated PV curves that were systematically altered by changes in RV, TLC, ADC, and $\gamma$. Mean values of $k$ at baseline and follow-up were also compared using ANOVA. For multiple comparisons, Fishers least significant difference post hoc test was used. In all statistical analyses, results were considered significant when the probability of making a type I error was $<5 \%(P<0.05)$.

\section{RESULTS}

Study subjects. Baseline demographic characteristics are provided in Table 2 for the 19 subjects enrolled (10 men) and show that the mean age and body mass index for each subgroup were not significantly different. As the COPD subjects and healthy volunteers were enrolled based on $\mathrm{FEV}_{1}$ and $\mathrm{FEV}_{1}$ / FVC, according to GOLD criteria (35), the mean values for $\mathrm{FEV}_{1}$ and $\mathrm{FEV}_{1} / \mathrm{FVC}$ for each subject subgroup reflect the

Table 4. Micromechanical model equation parameters

\begin{tabular}{lccc}
\hline \hline \multicolumn{1}{c}{ Parameter } & Healthy Volunteers & Stage II COPD & Stage III COPD \\
\hline$n$ & 5 & 8 & 6 \\
$\mathrm{~V}, \mathrm{~cm}^{3}$ & $3,060 \pm 430$ & $4,240 \pm 760$ & $6,270 \pm 1,770$ \\
$S, \times 10^{4} \mathrm{~cm}^{2}$ & $75 \pm 10$ & $91 \pm 21$ & $120 \pm 30$ \\
$N, \times 10^{8}$ alveoli & $3.9 \pm 0.5$ & $3.7 \pm 1.3$ & $4.3 \pm 0.6$ \\
$R, \mathrm{~mm}$ & $0.123 \pm 0.003$ & $0.142 \pm 0.011$ & $0.150 \pm 0.010$ \\
$R_{0}, \mathrm{~mm}$ & $0.108 \pm 0.004$ & $0.129 \pm 0.008$ & $0.141 \pm 0.012$ \\
$\gamma, \mathrm{dyn} / \mathrm{cm}$ & $18 \pm 4$ & $23 \pm 7$ & $34 \pm 9$ \\
\hline
\end{tabular}

Values are means $\pm \mathrm{SD} ; n$, no. of subjects; $\mathrm{V}$, volume; $N$, alveolar number; $R$, alveolar radius; $R_{0}$, radius at residual volume; $\gamma$, surface tension. All values, except $R_{0}$, are computed at a lung volume of $\mathrm{RV}+1$ liter.
GOLD criteria categorization; a one-way ANOVA showed these were significantly different between all subject groups. In addition, ANOVA showed that RV was significantly different for stage III COPD compared with healthy $(P<0.01)$; FRC was significant different for stage II and stage III COPD compared with healthy $(P<0.05)$. IC and TLC were decreased and increased, respectively, for both COPD cohorts compared with healthy elderly; however, neither change was statistically significant.

${ }^{3} \mathrm{He}$ MR images. Hyperpolarized ${ }^{3} \mathrm{He}$ MRI spin-density images (Fig. 3, Ai and Bi), ${ }^{3} \mathrm{He}$ ADC maps (Fig. 3, Aii and Bii), and corresponding ADC histograms (Fig. 3, Aiii and Biii) are shown for two representative subjects: one elderly healthy volunteer (subject $A$ ) and one subject with stage III COPD (subject $B$ ). For all subjects, and as previously described (10), a paired $t$-test showed that ADC values were significantly greater $(P<0.01)$ in the most anterior compared with the most posterior slices. The signal-to-noise ratio (SNR) for each slice was computed by taking the average signal intensity of a region in the lung slice and dividing by the standard deviation of the

Table 5. Results from micromechanical model

\begin{tabular}{lccc}
\hline \hline \multicolumn{1}{c}{ Parameter } & Healthy Volunteers & Stage II COPD & Stage III COPD \\
\hline$n$ & 5 & 8 & 6 \\
$R_{\text {alv }}, \mathrm{mm}^{2}$ & $0.150 \pm 0.010$ & $0.161 \pm 0.009$ & $0.164 \pm 0.004$ \\
$S / \mathrm{V}, \mathrm{cm}^{-1}$ & $200 \pm 13$ & $190 \pm 10$ & $183 \pm 5$ \\
$\gamma, \mathrm{dyn} / \mathrm{cm}$ & $30 \pm 6$ & $33 \pm 11$ & $41 \pm 8$ \\
$\mathrm{P}_{\text {tissue }}, \% \mathrm{Ptp}$ at TLC & $4.0 \pm 0.5$ & $2.8 \pm 0.6$ & $1.6 \pm 1.0$ \\
$\mathrm{P}_{\text {surface }} \% \mathrm{Ptp}$ at TLC & $20 \pm 10$ & $45 \pm 13$ & $68 \pm 20$ \\
$\mathrm{P}_{\text {duct, }} \% \mathrm{Ptp}$ at TLC & $75 \pm 10$ & $52 \pm 12$ & $30 \pm 19$ \\
$\mathrm{Ptp}, \mathrm{cmH}_{2} \mathrm{O}$ & $23 \pm 11$ & $10 \pm 4$ & $8 \pm 2$ \\
$k, \mathrm{cmH}_{2} \mathrm{O}^{-1}$ & $0.044 \pm 0.006$ & $0.080 \pm 0.009$ & $0.087 \pm 0.008$ \\
\hline \multicolumn{4}{c}{ Values are means $\pm \mathrm{SD} ; n$, no. of subjects; $\mathrm{R}_{\text {alv }}$, alveolar radius; $S / \mathrm{V}, S$-to-V } \\
ratio; $\mathrm{P}_{\text {tissue }}$, contribution of peripheral tissue network to transpulmonary \\
pressure (Ptp); $\mathrm{P}_{\text {surface, }}$ contribution of alveolar surface tension; $\mathrm{P}_{\text {duct }}$, contri- \\
bution of duct rings; $k$, shape factor from exponential analysis. All values, \\
except $k$, are reported at TLC and derived from the mean ADC value of five \\
center slices.
\end{tabular}


noise level $(\sigma)$ in a $20 \times 20$ pixel region of background. The SNR of the five center slices were averaged to get an average SNR for each subject. The average subject SNR was 35 and, therefore, unlikely to have affected ADC values.

Generation of $L$. Table 3 provides a list of mean center slice ADC, $D_{\mathrm{T}}, R, L$, and alveolar volume for each subject. $D_{\mathrm{T}}$ was computed from ADC using Eq. 6, and the $R$ was subsequently computed using Eqs. 2 and 7.

Generation of simulated PV curves. The values for the parameters used in the micromechanical model were computed based on $L$ and RV (Table 4). Both COPD subgroups showed increased $L$ compared with healthy elderly (one-way ANOVA, $P<0.01$, stage II COPD; $P<0.001$, stage III COPD), which is characteristic of emphysematous damage in COPD. $S$ and $\gamma(S)$ were significantly increased in stage III COPD compared with healthy subjects (one-way ANOVA, $P<0.01$ for both parameters). Table 5 shows the results provided by the micromechanical model.

PV curves for the two representative subjects shown in Fig. 3 (one healthy volunteer and one subject with stage III COPD) are provided in Fig. 4, as well as the contribution of each term in Eq. 8 to total Ptp (right plot, Fig. 4). The anterior-posterior Ptp gradient was calculated as the difference in Ptp (at TLC) between the most anterior and posterior slices and dividing the difference by the mean Ptp. This anterior-posterior Ptp gradient was decreased significantly in stage II COPD and stage III COPD subjects compared with healthy elderly (one-way ANOVA, $P<0.05$ and $P<0.01$, respectively). Figure 5 shows PV curves for all subjects using the mean ADC value of five center slices for the calculation of $L$. The value of $k$ was derived by least squares fitting of each PV curve to Eq. 10, and, in COPD, this was significantly increased compared with that in healthy volunteers (one-way ANOVA; $P<0.05$, stage II COPD; $P<0.01$, stage III COPD).

We also evaluated four input parameters to better understand their effect on the simulated Ptp curves, and these results are shown in Fig. 6. Specifically, RV, TLC, and mean ADC for a

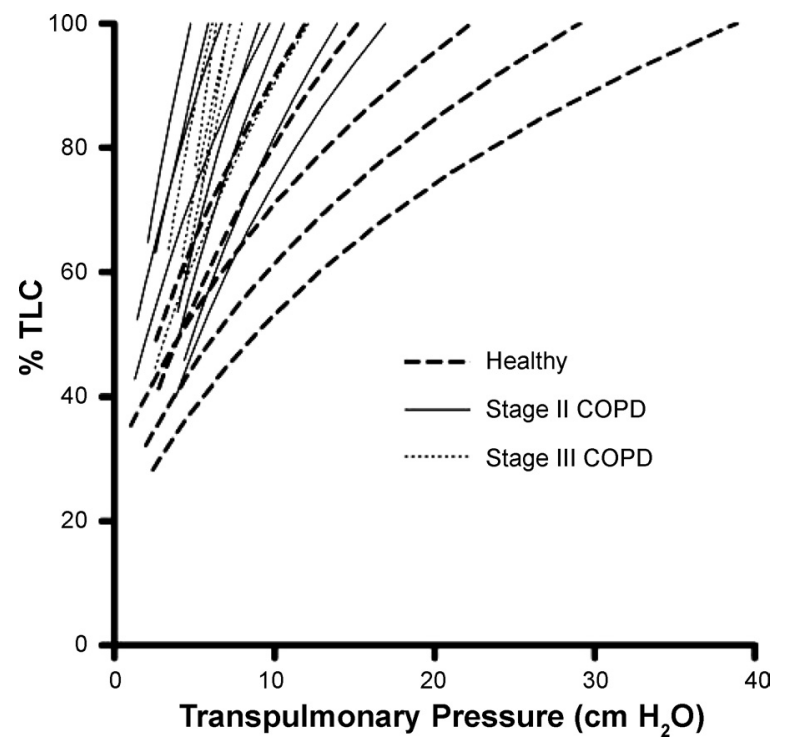

Fig. 5. Simulated PV curves for all subjects. PV curves for healthy volunteers (dashed line), stage II COPD (solid line), stage III COPD (dotted line) were generated from the mean ADC measurement of five center slices.

representative healthy subject are shown altered between -20 to $+20 \%$ to simulate significant changes in these values within the model; Ptp was calculated for each of these cases (Fig. 6, $A-C) . \gamma(S)$ was also varied by multiplying $\gamma(S)$ at each lung volume with a scaling constant (Fig. 6D). These results show that $20 \%$ changes in ADC $(P<0.0001)$, TLC $(P=0.02)$, and $\mathrm{RV}(P<0.0001)$ resulted in significant changes in the value of $k$. However, a $20 \%$ change in $\gamma(S)$ lead to no significant change in $k(P=0.37)$. It is important to note that these changes represent theoretical error in the model that are greater than the $95 \%$ confidence intervals for measurement error that we previously measured (i.e., precision for TLC and RV using coefficients of variation ranging were $1-3 \%$ and ADC coefficients of variation $<0.5 \%$ ).
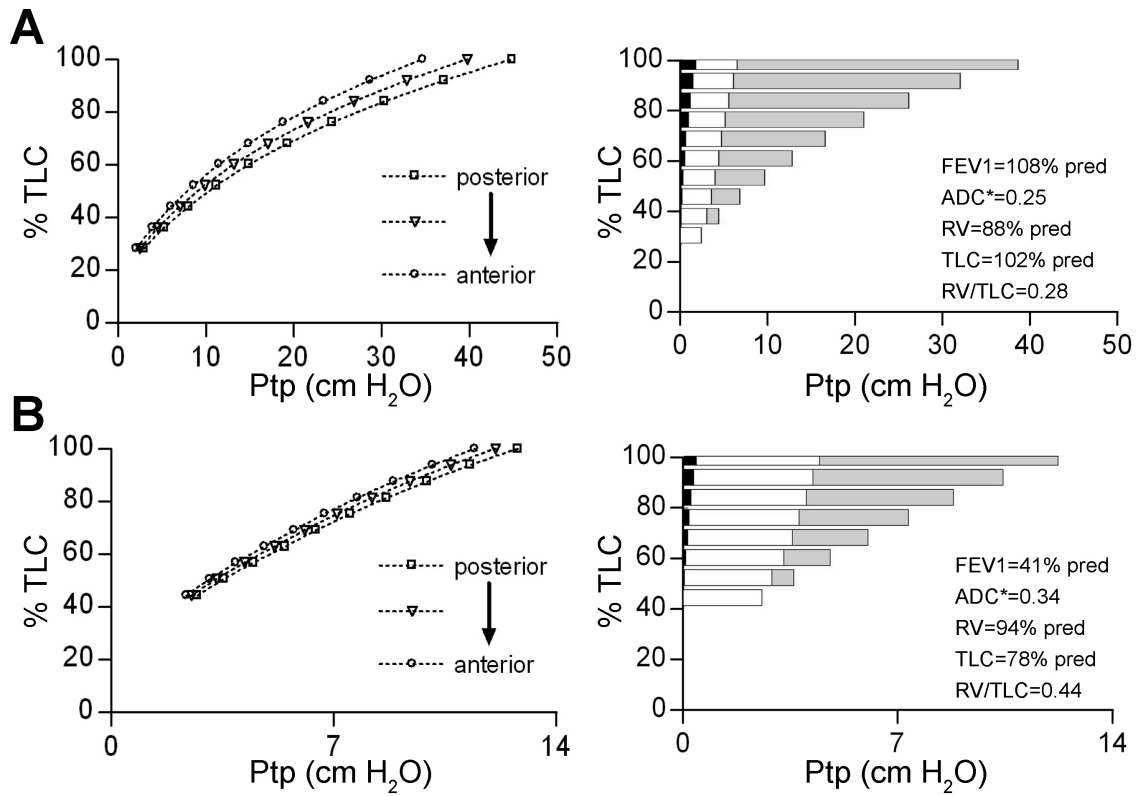

$\mathrm{ADC}^{*}=$ mean $\mathrm{ADC}$ of 5 center slices

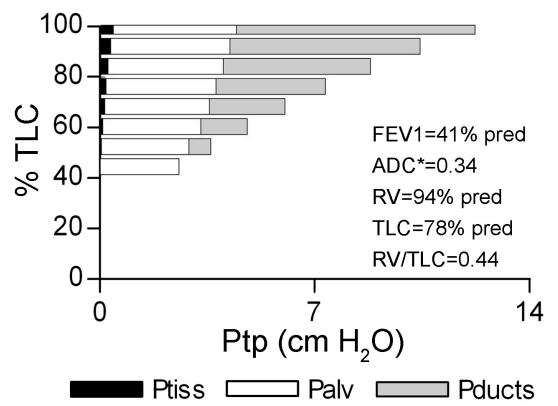

Fig. 4. Representative transpulmonary pressure (Ptp)volume (PV) curves. PV curves for the same subjects shown in Fig. 3 ( $A$ : healthy elderly subject; $B$ : stage III COPD subject) were generated for each of five center slices using Eq. 8 (left). The histogram (right) shows the relative contribution of each term in Eq. 8 to Ptp. TLC, total lung capacity; $\mathrm{FEV}_{1}$, forced expiratory volume in 1 $\mathrm{s}$; RV, residual volume; Ptiss, tissue pressure; Palv, alveolar pressure; Pducts, duct pressure. 
Fig. 6. Testing the model. PV curves were generated for a single healthy elderly volunteer using $\mathrm{ADC} \pm 20 \%(A), \mathrm{RV} \pm 20 \%(B), \mathrm{TLC} \pm 20 \%$ $(C)$, and surface tension $(\gamma) \pm 20 \%(D)$.
A

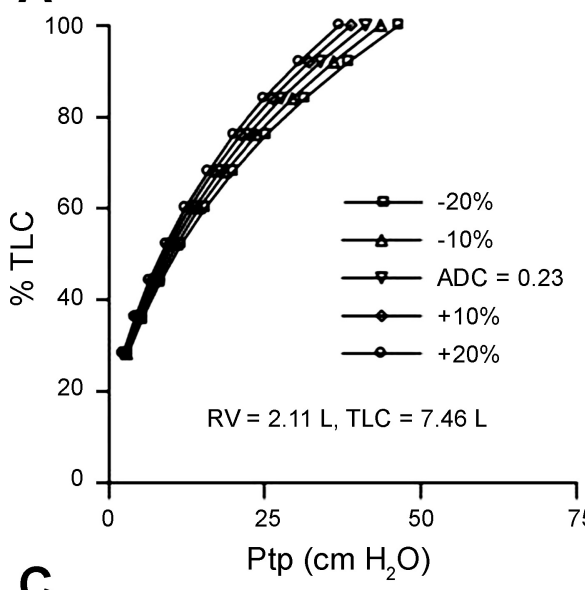

100

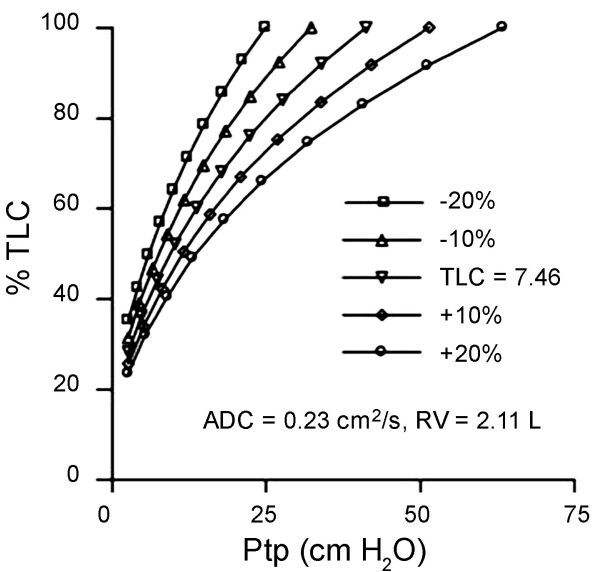

B
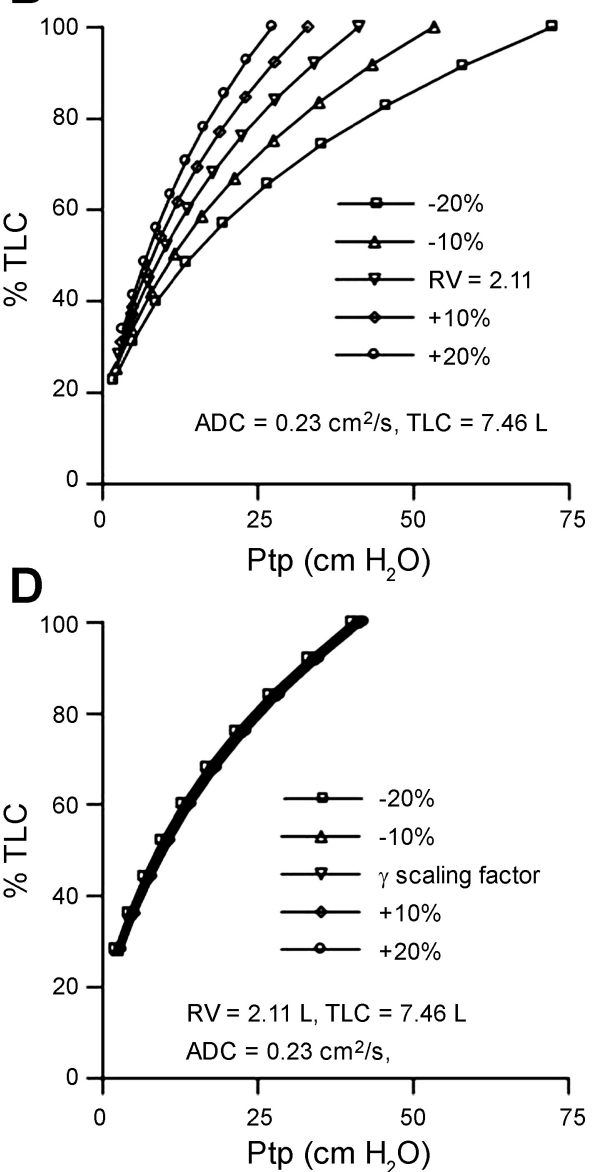

In Fig. 7, simulated PV curves are provided for three representative subjects who returned $\sim 2$ yr later for follow-up [Fig. 7, $A i$ (healthy volunteer), $B i$ (stage II COPD), and $C i$ (stage III COPD)], reflecting the changes in mean ADC, RV, and TLC that occurred during that time period for each subject. The accompanying histograms illustrating the contribution of each term in Eq. 8 are also shown for each subject at baseline (Fig. 7, Aii-Cii) and follow-up (Fig. 7, Aiii-Ciii). Baseline and follow-up $k$ values by subject group are provided in Table 6 and showed no significant change; however, the $S$-to-volume ratio was significantly decreased for stage II COPD patients at follow-up (paired $t$-test, $P=0.03$ ).

Effects of model assumptions on PV curves. Several assumptions were made to derive PV curves, and we have evaluated the sensitivity of the model to these assumptions. Similar to the previous section, we set the model input parameters to representative values for a healthy subject and vary several key parameters to measure their effect on the shape factor $k$.

The $D_{\mathrm{L}} / D_{\mathrm{T}}$ was approximated as 3 to derive $E q$. 6; however, there is some physiological variability in healthy subjects and patients with severe emphysema, as demonstrated by Yablonskiy et al. (48). Based on that work, we altered $D_{\mathrm{L}} / D_{\mathrm{T}}$ from 2.5 to 4.0 , which represent the most extreme values of the $D_{\mathrm{L}} / D_{\mathrm{T}}$ that were presented. The ratio of the $L$ to $R(L / R)$ was also assumed to be constant in our model, with a value of 0.333 , while work by Sukstanskii and Yablonskiy (39) estimated that $L / R$ could vary by $20 \%$ in healthy and slightly emphysematous human lungs. Accordingly, we varied $L / R$ by $\pm 20 \%$. In $E q .8$, we assume that each alveoli can be represented as a sphere, and that the number of duct ring fibers $n$ is equal to the number of alveoli $N$. We tested the assumption of spherical alveoli by varying the shape to a three-fourth sphere or one-half sphere. The relation between $n$ and $N$ was varied arbitrarily by $20 \%$.

Numerical results of model sensitivity to these assumptions are summarized in Table 7. The model was insensitive to changes in the $D_{\mathrm{L}} / D_{\mathrm{T}}$ and deviated by $-10.9 \%$ when $D_{\mathrm{L}} / D_{\mathrm{T}}$ was set to 4 . Conversely, the model was extremely sensitive to changes in $L / R$, with a $20 \%$ reduction in $L / R$ producing a $43.6 \%$ reduction in $k$. Altering the alveoli shape had a large effect as well, and shifting from a spherical model to a hemispherical model decreased $k$ by $45.5 \%$, although shifting to a three-fourth spherical model only decreased $k$ by $18.9 \%$. Finally, the model was moderately sensitive to changes in the ratio $n / N$, with a $20 \%$ increase and decrease in the ratio causing a $15.8 \%$ decrease and $18.8 \%$ increase in $k$, respectively.

Effects of heterogeneity on model results. The micromechanical model operates on the assumption that each physiological parameter can be estimated by a single average value, and that the entire lung can be represented by average parameters. However, in emphysema, there are regional differences in tissue properties, which may affect the simulated whole lung PV curve. To test for the effects of this heterogeneity, we divided the center slice of a representative COPD stage III subject into four compartments and eight compartments 
Ai

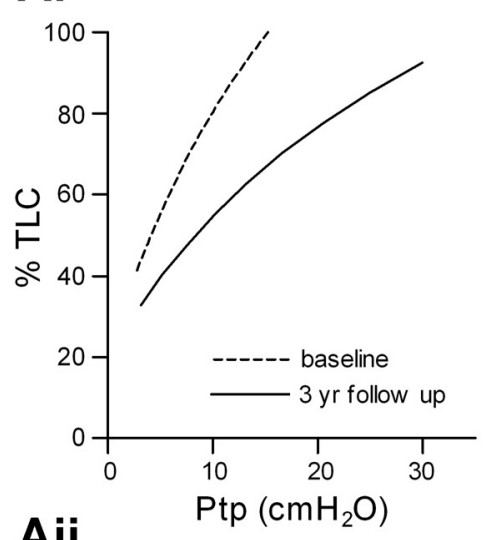

Aii

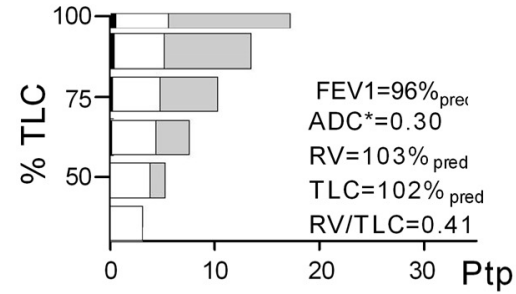

Aiii

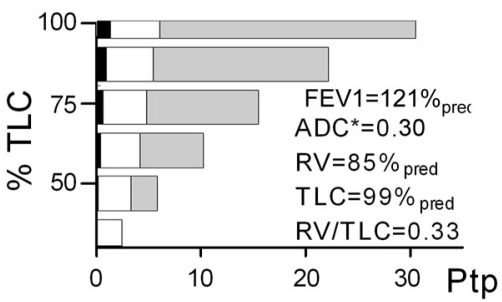

Bi Stage II COPD

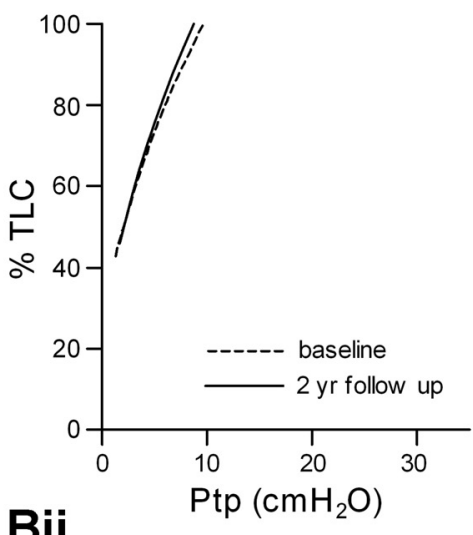

$\mathrm{Bii}$

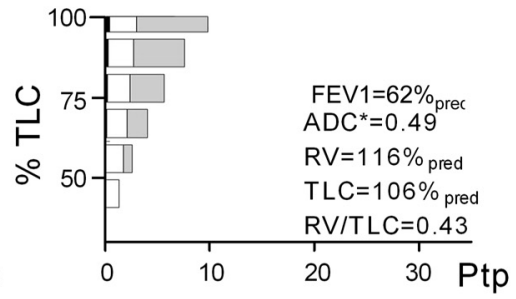

Biii

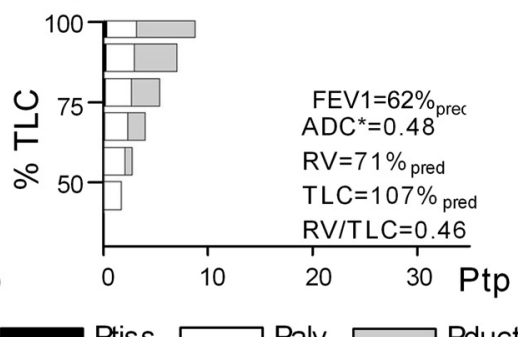

Ci Stage III COPD

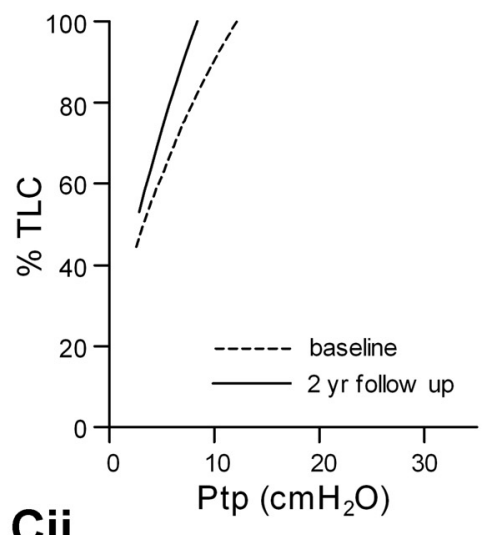

Cii

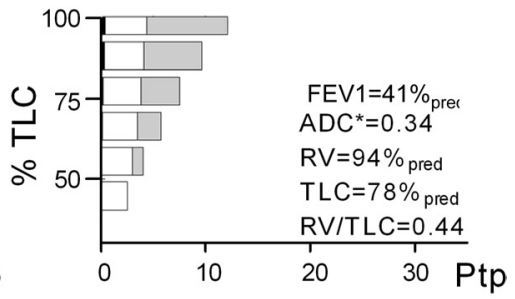

Ciii

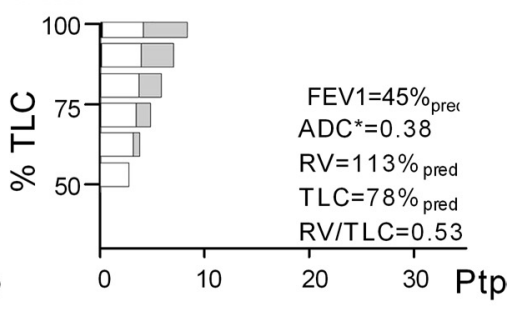

$A D C^{*}=$ mean $A D C$ of 5 center slices

Ptiss

Fig. 7. Longitudinal PV curves. PV curve plots $(i)$ illustrate changes in Ptp for each subject from baseline to follow-up. Histograms were generated for baseline (ii) and follow-up (iii). A: healthy; $B$ : stage II COPD; $C$ : stage III COPD.

(Fig. 8). Local physiological parameters and PV curves were generated independently for each compartment. Mean ADC in each compartment differed slightly compared with the mean $\mathrm{ADC}$ of the entire center slice, with the average deviation being $4.5 \%$ for four compartments and $5.7 \%$ for eight compartments.

PV curves were generated independently for each compartment (Fig. 9, shaded areas), and also for the center slice using averaged parameters. PV curves for individual compartments were found to differ slightly from the center slice PV curve; however, the $k$ value of any compartment did not deviate by $>3 \%$ from the $k$ value of the center slice PV curve. The average PV curve of all of the compartments was nearly identical to the center slice PV curve (Fig. 9).

\section{DISCUSSION}

In this small pilot study of COPD ex-smokers and healthy age-matched never-smokers, we aimed to develop a way to generate transpulmonary PV curves based on micromechanical models of the lung, plethysmography measurements of lung volumes, and ${ }^{3} \mathrm{He}$ MRI ADC values. Accordingly, we report the following: 1) the use of ${ }^{3} \mathrm{He}$ MRI ADC to estimate average alveolar dimensions in specific ROI; 2) application of ADC mea-

Table 6. Comparison of ${ }^{3} \mathrm{He}$ MRI-derived PV curve parameters at baseline and follow-up

\begin{tabular}{lcccr}
\hline \hline & & \multicolumn{2}{c}{ Baseline } & \multicolumn{1}{c}{ Follow-up } \\
\cline { 2 - 4 } & $\mathrm{n}$ & $k, \mathrm{cmH}_{2} \mathrm{O}^{-1}$ & $S / \mathrm{V}, \mathrm{ml}^{-1}$ & $S / \mathrm{V}, \mathrm{ml}^{-1}$ \\
\hline Healthy elderly & 3 & $0.049 \pm 0.013$ & $204 \pm 15$ & $0.030 \pm 0.006$ \\
Stage II COPD & 7 & $0.079 \pm 0.027$ & $187 \pm 10$ & $0.084 \pm 0.016$ \\
Stage III COPD & 5 & $0.083 \pm 0.019$ & $182 \pm 5$ & $0.090 \pm 0.020$ \\
\hline
\end{tabular}

Values are means $\pm \mathrm{SD} ; n$, no. of subjects. $S / \mathrm{V}$ is at TLC. 
Table 7. Sensitivity of model results to key parameters

\begin{tabular}{cc}
\hline \hline Parameter & $\Delta k, \%$ \\
\hline Normal: $D_{\mathrm{L}} / D_{\mathrm{T}}=3$ & \\
$D_{\mathrm{L}} / D_{\mathrm{T}}=2.5$ & +8.3 \\
$D_{\mathrm{L}} / D_{\mathrm{T}}=4$ & -10.9 \\
Normal: $L / R=0.333$ & \\
$L / R=0.27$ & -43.6 \\
$L / R=0.40$ & +35.8 \\
Normal: spherical alveoli & \\
Three-fourths spherical & -18.9 \\
One-half spherical & -45.5 \\
Normal: $n=N$ & \\
$n=0.8 \mathrm{~N}$ & +18.8 \\
$n=1.2 \mathrm{~N}$ & -15.8 \\
\hline
\end{tabular}

$\Delta k, \%$, Percent change in $k$ from normal. $D_{\mathrm{L}} / D_{\mathrm{T}}$, ratio of longitudinal diffusion coefficient to transverse diffusion coefficient; $L / R$, ratio of $L$ to $R ; n$, number of duct rings; $N$, number of alveoli.

surements in an established lung tissue micromechanical model to generate regional transpulmonary PV curves; 3) comparison of transpulmonary PV curves for specific ROIs in healthy elderly and COPD subjects; and 4) comparison of baseline and follow-up PV curves for subjects who returned $\sim 2$ yr later. To our knowledge, this is the first application of hyperpolarized ${ }^{3} \mathrm{He} \mathrm{ADC}$ for the generation of noninvasive transpulmonary PV curves with the evaluation of ROI and longitudinal differences.

First, we derived the number of alveoli and mean alveolar dimension from ADC measurements following the work of Yablonskiy et al. (48). ${ }^{3} \mathrm{He}$ images were likely not biased due to low SNR and, therefore, the resulting ADC measurements likely reflected disease severity and not noise level (31). We observed significant differences in alveolar dimensions between healthy subjects and those with stage II and stage III COPD; however, as shown in Table 3, alveolar number was not significantly different for either COPD group compared with the healthy volunteers. This observation may be partly explained by the variability of ADC values across COPD subjects, suggesting that, within a single GOLD group, there are different disease processes that reduce lung function without concomitantly reducing alveolar number (e.g., chronic bronchitis). Another possible explanation is related to the diffusion time used in this study; acinar duct dimensions were likely probed, and, to estimate $L$, we assumed a constant relationship between $L$ and $R$, regardless of disease state, although this specific relationship was previously derived from normal lung (21).

Second, using $L$ dimensions in the Stamenovic model, we generated transpulmonary PV curves, and, as might be ex- pected, there were significant differences in PV curves for healthy and COPD subjects. $\gamma(S)$ also appeared to provide a greater proportion of recoil pressure in COPD subjects (Fig. 4), and, as shown in Table $5, \gamma(S)$ contributed to $\sim 20 \%$ of the recoil pressure for healthy subjects, $45 \%$ for stage II COPD subjects, and $68 \%$ for stage III COPD subjects. This result is in agreement with previous work of Ingenito and colleagues (21), who reported that tissue recoil forces decreased rapidly in emphysema, while $\gamma(S)$ remained an important contributor to Ptp. The increase in $\gamma(S)$ derived for the COPD subjects in our study was directly related to the increase in total $S$ (Table 4 ), which is contrary to results reported by Ingenito et al. that demonstrated a decrease in $S$ in COPD. One explanation for this discordant result is that, in the present study, $S$ was derived from the micromechanical equations, and that its calculation was more strongly influenced by the degree of hyperinflation reported for COPD subjects compared with the decreased $S$ predicted by the high ADC values that reflected enlarged air spaces in the COPD patients. Similarly, surface area-to-volume ratio values predicted from our simulations were larger than those reported by Ingenito et al.

It is important to note that $k$ values predicted from our simulated PV curves were lower than those previously proposed by Ingenito et al. (21). One contributing factor to the apparently lower $k$ values we report in this study may be attributed to the diminished simulated alveolar tissue recoil force, which represented a significant portion of Ptp in the previous analysis (21). The loss of this portion of recoil pressure flattens the simulated PV curves we present here, which subsequently lowers $k$. Another noteworthy difference between our result and previous work (21) is that $\gamma(S)$ was not equal to $0 \mathrm{dyn} / \mathrm{cm}$ at $\mathrm{RV}$, perhaps reflecting the significant gas trapping for the COPD patients in our study that may have lead to residual recoil pressure at $\mathrm{RV}$.

Third, we showed significant differences in Ptp gradients in the anterior-posterior direction (Fig. 4), which may reflect the postural changes in ADC previously described (10). We also observed that both COPD cohorts had significantly reduced gradients compared with a healthy age-matched cohort $(P<$ 0.01 , stage II COPD and $P<0.001$, stage III COPD). We have previously reasoned that, in COPD subjects, gas trapping in the posterior portion of the lung makes the air spaces less compressible, which reduced the anterior-posterior gradient compared with healthy volunteers. It was previously established in a number of studies $(8,11)$ that higher lung inflation levels increase ADC values. This effect is also observed in COPD subjects, where gas-trapped posterior lung regions have higher ADC values than what might be predicted. With gas
Fig. 8. Mean ADC and standard deviation for a center slice and each compartment. Mean ADC values are given in $\mathrm{cm}^{2} / \mathrm{s}$, and standard deviations (in parentheses) are reported for the center slice and each compartment of a single COPD stage III subject.

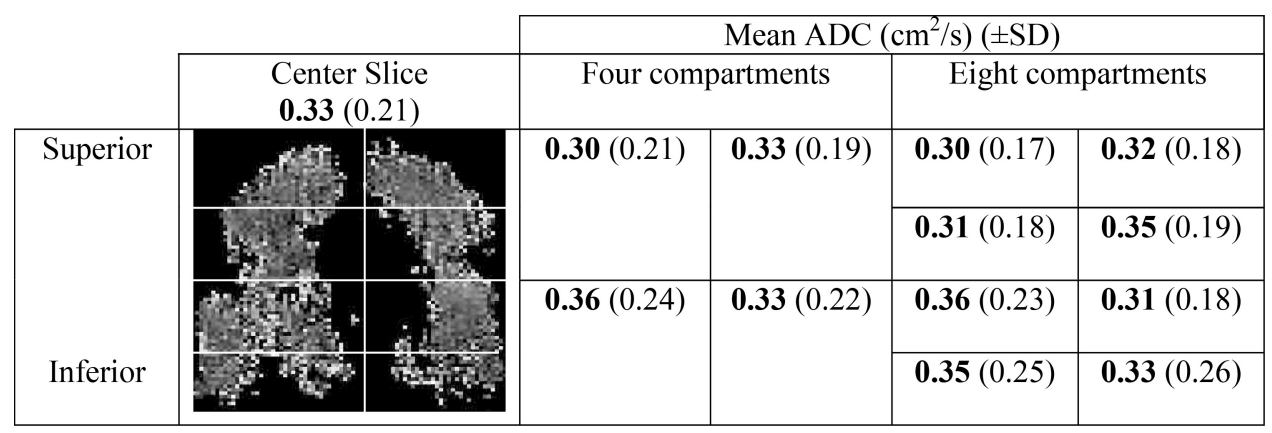




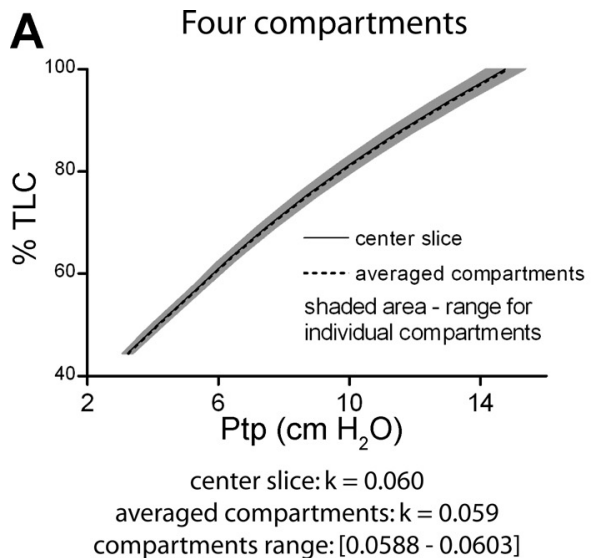

compartments range: [0.0588 - 0.0603]

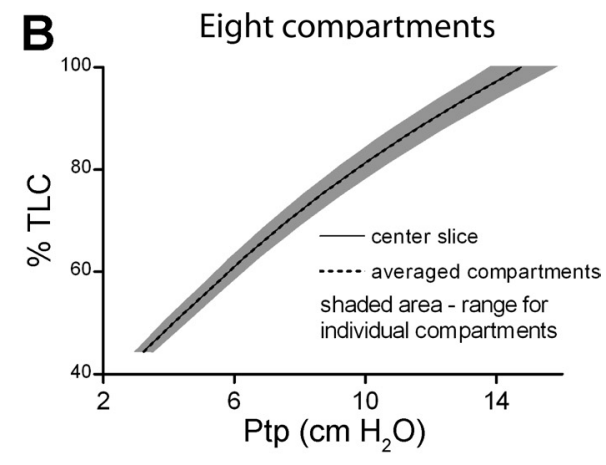

center slice: $\mathrm{k}=0.060$

averaged compartments: $\mathrm{k}=0.060$

compartments range: [0.0583 - 0.0605]
Fig. 9. Compartment heterogeneity effects on simulated PV curve. PV curves are generated for the center slice and compartments of a single stage III COPD subject and fitted to a monoexponential function (Eq. 10). PV curves were generated for four compartments $(A)$ and eight compartments $(B)$. The shape factor $k$ is reported for the center slice PV curve and the average PV curve of the compartments. The range of compartment PV curves is shown (shaded), and the maximum and minimum $k$ are also reported. trapping, elevated recoil pressures would result in decreased Ptp gradients.

Finally, we also demonstrated the utility of ${ }^{3} \mathrm{He}$ MRIderived PV curves for longitudinal analysis (Fig. 7) and showed that changes in ADC, RV, and TLC for some subjects resulted in significant alterations in surface area-to-volume ratio. We also expect that, with a greater number of longitudinal study subjects, significant changes in $k$ may be detected. When comparing longitudinal PV curves, however, it is important to note that parameter analysis (Fig. 6) showed the sensitivity of the curves to RV and TLC, pointing out the importance of consistency in longitudinal plethysmography measurements. The change in PV curves for one healthy subject (Fig. 7A) is likely due to this sensitivity. This particular patient had a large decrease in RV/TLC from baseline to follow-up (0.41 to 0.33 ), which would cause a large increase in Ptp. Nonphysiological differences in lung inflation were controlled by having each subject rehearse the breath-hold maneuver while seated outside the MR scanner and once while inside the scanner.

We recognize and acknowledge a number of important limitations of this work. Compared with previous work (21) that demonstrated that the peripheral tissue network contributed $>50 \%$ of Ptp in normal subjects and at least $20 \%$ in emphysema, the contribution of the peripheral tissue network to Ptp is likely underestimated (4\% for healthy elderly and $2.8 \%$ for COPD) in our analysis. We expected that, in accordance with this previous work, peripheral tissue would contribute to almost one-half of the pressure in the lung, especially for the healthy volunteers with intact peripheral lung tissue, which allows for a large expansion from RV to TLC, and furthermore allows the peripheral lung tissue to stretch and generate recoil. However, none of the COPD subjects in this study showed such large lung expansion, and even the youngest never-smoker (aged $58 \mathrm{yr}$ at baseline) reported a RV/TLC of $0.28(\mathrm{RV}=2$ liters, $\mathrm{TLC}=7$ liters $)$.

This may have resulted because of the small recoil pressures due to the minimal stretching of the peripheral fibers (calculated recoil forces $=5.5 \mathrm{dyn}$ in our dataset), creating a maximum recoil pressure of $\sim 2.2 \mathrm{cmH}_{2} \mathrm{O}$. One possible improvement to the model is to include the recoil force generated by the pleural membrane into the peripheral tissue system, and we note that Hajji et al. (19) previously reported that the tension in the pleural membrane contributed $\sim 20 \%$ of the work performed by the lung during deflation. We also recognize that the relationship between $\gamma(S)$ and $S$ in COPD is not well understood. While it is commonly recognized that the $\gamma(S)-S$ curve changes nonlinearly with inspiration vs. expiration, we used an approximate linear relationship to represent the $\gamma(S)-S$ curve. In addition, using a single $\gamma(S)-S$ relationship for both healthy and COPD lungs may not be appropriate, because this implies that the slow expansion of an alveolus in emphysema might be similar to a single stretch of an alveolus during respiration, but the two are likely somewhat different physiological processes. To our knowledge, there have been no studies that experimentally measure $\gamma(S)-S$ curves for healthy elderly with comparison to COPD subjects, and characterization of changes in the intrinsic properties of surfactant in disease is incomplete. It is worth noting, however, that recent work has shown no change in surfactant in emphysematous elastase-treated mice (30). In the future, it will be important to account for interdisease and intersubject differences in $\gamma(S)$. Another limitation that is important to note is the fact that ADC values only reflect those regions of the lung that are ventilated, which limits the utility of the ADC measurement to only those areas of the lung that can be ventilated during the timeframe of the MR scan. Those regions that cannot be ventilated due to bullae, gas trapping with long time constants, or closed or narrowed airways do not report ADC values, which certainly is a limitation of this approach in subjects with very poor ventilation.

A number of assumptions in the model and the selection of model parameters certainly affect our results and warrant discussion, as shown in Table 7. The first assumption was to set $D_{\mathrm{L}} / D_{\mathrm{T}}$ to 3 for all subjects. This approximation was necessary, since image data were only acquired at two $b$ values (0 and 1.6), and ADC was computed using a monoexponential model. In the multi- $b$ value method proposed by Yablonskiy et al. (48), the mean and anisotropic ADCs can be calculated to deduce both $D_{\mathrm{L}}$ and $D_{\mathrm{T}}$. Despite this simplistic assumption and ignoring diffusion anisotropy, the physiological variability of $D_{\mathrm{L}} / D_{\mathrm{T}}$ only weakly affected the resulting PV curve. The shape factor $k$ deviated by $10.9 \%$ in the worst case estimated of $D_{\mathrm{L}} / D_{\mathrm{T}}$ (Table 7). Furthermore, $D_{\mathrm{T}}$ is relatively insensitive to changes in $b$ value (39), thus there is minimal error in $D_{\mathrm{T}}$ due to its calculation using only two small $b$ values. We had also assumed that the $L / R$ was constant, regardless of disease status, which may not be true for severely emphysematous lungs. This approximation could introduce relatively large error in the resulting PV 
curve; a $20 \%$ decrease in $L / R$ decreased $k$ by $43.6 \%$ (Table $7)$. This limitation can be overcome in the future by acquiring MR images at several $b$ values to better estimate $r, R, L$, $D_{\mathrm{T}}$, and $D_{\mathrm{L}}$ (39). Finally, the assumptions that alveoli remain spherical in COPD and that the number of duct rings $n$ remain equivalent to the number of alveoli $N$ are both debatable and strongly influence the resulting PV curve. For example, assuming the alveoli are all hemispherical reduces $k$ by $45.5 \%$, and a change in the $n$-to- $N$ ratio by $20 \%$ changes $k$ by $16-19 \%$ (Table 7). Unfortunately, both of these parameters likely change from subject to subject and cannot be determined a priori or estimated using in vivo ${ }^{3} \mathrm{He}$ imaging.

A final limitation with the model is that it fails to account for heterogeneity in lung structure and micromechanics. In severe emphysematous lungs, which are characterized as having a wide range of ADC values and alveolar dimensions, it becomes contentious whether a mean ADC value is truly representative of the entire lung. We tested the representativeness of a mean global ADC by comparing the PV curve generated at a single slice level in a stage III COPD subject with PV curves generated at a compartment level in the same slice (Figs. 8 and 9). The resulting compartment PV curves differed in their shape factor $k$ by $<3 \%$ compared with the whole slice PV curve, despite differences between compartment ADC and whole slice ADC of up to $10 \%$. Additionally, the average PV curve among all of the compartments is nearly identical to the whole slice PV curve. This result suggests that simulated whole lung PV curves are insensitive to large-scale heterogeneity.

While regional PV curves can be generated to capture regional differences in lung mechanics, the added issue of ADC sensitivity to small-scale heterogeneity is raised. Computing PV curves at a pixel level is problematic, as Jacob et al. (22) had demonstrated that, at the single-pixel level, ADC is sensitive to heterogeneity in microscopic structure rather than true changes in alveolar dimensions. However, that group used a pixel size of $0.5 \mathrm{~mm} \times 0.5 \mathrm{~mm}$, with a slice thickness of 2 $\mathrm{mm}$, while our pixel size was $3.125 \mathrm{~mm} \times 3.125 \mathrm{~mm}$, with a slice thickness of $30 \mathrm{~mm}$, which might mitigate small-scale heterogeneity effects. The same group also found that mean ADC at larger scale correlated well with mean alveolar dimensions, and that the Yablonskiy et al. model (48) could adequately describe the emphysematous lung. Previous work in our laboratory (11) has also demonstrated that heterogeneity can exist at a regional pixel level, which is not accounted for in this present work. A remaining question is what effect does regional heterogeneity have on image-derived PV curves.

In future studies, it will be important to directly compare simulated PV curves for a larger group of COPD and healthy volunteers with PV curves acquired experimentally to better understand the regional information we are simulating. At the time of study, our laboratory did not have access to the necessary equipment nor expertise to experimentally measure PV curves in these same subjects using the transesophageal balloon method. Conducting these measurements years after the original scan would introduce a discrepancy between the simulated and measured PV curve, as the patient's lung function may have altered in that time. However, when comparing our derived PV curves to typical measured PV curves, we do note that our derived PV curves have much lower $k$ values, implying that these PV curves do not plateau as quickly as measured ones. It may be the case that derived PV curves only accurately describe measured PV curves at low volumes. At the same time, it is possible that imaging-derived regionally based PV curves might provide a way to better characterize heterogenous or focal disease, since information is captured from all regions of the lung and the contributions can be evaluated independently. In other words, the transesophageal balloon method measures Ptp contributions from the entire lung adjacent to the lung pleural cavity. By comparing both experimental and imaged-derived PV curves in cases with minimal heterogeneity, such as in healthy lungs, and in cases marked by large regional heterogeneity, such as in emphysematous lungs, it would be possible to empirically determine the effects of heterogeneity on lung mechanics.

The current result, however, demonstrated that hyperpolarized ${ }^{3} \mathrm{He}$ ADC can be used to derive alveolar dimensions on a regional basis and generate transpulmonary PV curves using the Stamenovic model of lung mechanics. For healthy elderly and COPD subjects, Ptp was greater in the posterior region of the lung than in the anterior region, and significant differences were detected between subject groups. The results of this preliminary analysis suggest that hyperpolarized ${ }^{3} \mathrm{He}$ MRI may provide an important noninvasive method for studying regional transpulmonary PV curves in COPD.

\section{ACKNOWLEDGMENTS}

We thank Drs. P. Macklem and E. Ingenito for helpful discussions that greatly improved this work and manuscript. We are grateful to S. Halko and S. McKay for pulmonary function testing, clinical coordination, and clinical database management; A. Wheatley and A. Farag for production and dispensing of ${ }^{3} \mathrm{He}$ gas; and C. Harper-Little for MRI of research subject volunteers. The use of an onsite hyperpolarized ${ }^{3} \mathrm{He}$ gas polarizer system [HeliSpin, General Electric Health Care (GEHC), Durham, NC] was provided to Robarts Research Institute through an agreement with GEHC.

\section{GRANTS}

S. Choy gratefully acknowledges stipend support from the Summer Research Training program, Schulich School of Medicine and Dentistry (The University of Western Ontario, London Canada). Funding support from the Canadian Institutes of Health Research and Canadian Lung Association is also gratefully acknowledged.

\section{DISCLOSURES}

No conflicts of interest, financial or otherwise, are declared by the author(s).

\section{REFERENCES}

1. Albert MS, Balamore D. Development of hyperpolarized noble gas MRI. Nucl Instrum Methods Phys Res A 402: 441-453, 1998.

2. Bachofen H, Hildebrandt J, Bachofen M. Pressure-volume curves of air- and liquid-filled excised lungs-surface tension in situ. J Appl Physiol 29: 422-431, 1970.

3. Chen XJ, Moller HE, Chawla MS, Cofer GP, Driehuys B, Hedlund LW, Johnson GA. Spatially resolved measurements of hyperpolarized gas properties in the lung in vivo. I. Diffusion coefficient. Magn Reson Med 42: 721-728, 1999

4. Choudhri A, Altes TA, Stay R, Mugler JP, II, de Lange EE. The occurrence of ventilation defects in the lungs of healthy subjects as demonstrated by hyperpolarized helium-3 MR imaging. In: Proceedings of the 93rd Scientific Assembly and Annual Meeting of the Radiolopgical Society of North America SSA21-05, 2007. Oak Brook, IL: RSNA, 2007.

5. Coxson HO, Mayo JR, Lam S, Santyr GE, Parraga G, Sin D. New and current clinical imaging techniques to study chronic obstructive pulmonary disease. Am J Respir Crit Care Med 180: 588-597, 2009.

6. Coxson HO, Mayo JR, Behzad H, Moore BJ, Verburgt LM, Staples CA, Pare PD, Hogg JC. Measurement of lung expansion with computed tomography and comparison with quantitative histology. J Appl Physiol 79: $1525-1530,1995$. 
7. de Lange EE, Mugler JP III, Brookeman JR, Knight-Scott J, Truwit JD, Teates CD, Daniel TM, Bogorad PL, Cates GD. Lung air spaces: MR imaging evaluation with hyperpolarized $3 \mathrm{He}$ gas. Radiology 210: 851-857, 1999.

8. Diaz S, Casselbrant I, Piitulainen E, Pettersson G, Magnusson P, Peterson B, Wollmer P, Leander P, Ekberg O, Akeson P. Hyperpolarized $3 \mathrm{He}$ apparent diffusion coefficient MRI of the lung: reproducibility and volume dependency in healthy volunteers and patients with emphysema. J Magn Reson Imaging 27: 763-770, 2008.

9. Eidelman DH, Ghezzo H, Bates JH. Exponential fitting of pressurevolume curves: confidence limits and sensitivity to noise. J Appl Physiol 69: $1538-1541,1990$.

10. Evans A, McCormack D, Ouriadov A, Etemad-Rezai R, Santyr G, Parraga G. Anatomical distribution of (3)He apparent diffusion coefficients in severe chronic obstructive pulmonary disease. J Magn Reson Imaging 26: 1537-1547, 2007.

11. Evans A, McCormack DG, Santyr G, Parraga G. Mapping and quantifying hyperpolarized $3 \mathrm{He}$ magnetic resonance imaging apparent diffusion coefficient gradients. J Appl Physiol 105: 693-699, 2008.

13. Fain SB, Altes TA, Panth SR, Evans MD, Waters B, Mugler JP III, Korosec FR, Grist TM, Silverman M, Salerno M, Owers-Bradley J. Detection of age-dependent changes in healthy adult lungs with diffusionweighted 3He MRI. Acad Radiol 12: 1385-1393, 2005.

14. Fain SB, Panth SR, Evans MD, Wentland AL, Holmes JH, Korosec FR, O'Brien MJ, Fountaine H, Grist TM. Early emphysematous changes in asymptomatic smokers: detection with $3 \mathrm{He}$ MR imaging. Radiology 239: 875-883, 2006

15. Fichele S, Woodhouse N, Swift AJ, Said Z, Paley MN, Kasuboski L, Mills GH, Van Beek EJ, Wild JM. MRI of helium-3 gas in healthy lungs: posture related variations of alveolar size. J Magn Reson Imaging 20: 331-335, 2004.

16. Fukaya H, Martin CJ, Young AC, Katsura S. Mechanial properties of alveolar walls. J Appl Physiol 25: 689-695, 1968.

17. Gierada DS, Woods JC, Bierhals AJ, Bartel ST, Ritter JH, Choong CK, Das NA, Hong C, Pilgram TK, Chang YV, Jacob RE, Hogg JC, Battafarano RJ, Cooper JD, Meyers BF, Patterson GA, Yablonskiy DA, Conradi MS. Effects of diffusion time on short-range hyperpolarized (3)He diffusivity measurements in emphysema. J Magn Reson Imaging 30: 801-808, 2009.

18. Haefeli-Bleuer B, Weibel ER. Morphometry of the human pulmonary acinus. Anat Rec 220: 401-414, 1988.

19. Hajji MA, Wilson TA, Lai-Fook SJ. Improved measurements of shear modulus and pleural membrane tension of the lung. $J$ Appl Physiol 47: 175-181, 1979.

20. Hoppin FGJ, Hildebrandt J. Mechanical properties of the lung. In: Bioengineering Aspects of the Lung, edited by West JB. New York: Dekker, 1977, p. 83-162.

21. Ingenito EP, Tsai LW, Majumdar A, Suki B. On the role of surface tension in the pathophysiology of emphysema. Am J Respir Crit Care Med 171: 300-304, 2005.

22. Jacob RE, Minard KR, Laicher G, Timchalk C. 3D 3He diffusion MRI as a local in vivo morphometric tool to evaluate emphysematous rat lungs. J Appl Physiol 105: 1291-1300, 2008.

23. Kauczor HU, Ebert M, Kreitner KF, Nilgens H, Surkau R, Heil W, Hofmann D, Otten EW, Thelen M. Imaging of the lungs using 3He MRI: preliminary clinical experience in 18 patients with and without lung disease. J Magn Reson Imaging 7: 538-543, 1997.

24. Kauczor HU, Ley-Zaporozhan J, Ley S. Imaging of pulmonary pathologies: focus on magnetic resonance imaging. Proc Am Thorac Soc 6: 458-463, 2009

25. Lai-Fook SJ, Rodarte JR. Pleural pressure distribution and its relationship to lung volume and interstitial pressure. J Appl Physiol 70: 967-978, 1991

26. Ley S, Zaporozhan J, Morbach A, Eberle B, Gast KK, Heussel CP, Biedermann A, Mayer E, Schmiedeskamp J, Stepniak A, Schreiber WG, Kauczor HU. Functional evaluation of emphysema using diffusion-weighted $3 \mathrm{He}-$ lium-magnetic resonance imaging, high-resolution computed tomography, and lung function tests. Invest Radiol 39: 427-434, 2004.

27. Ley-Zaporozhan J, Ley S, Kauczor HU. Proton MRI in COPD. COPD 4: 55-65, 2007.

28. Moller HE, Chen XJ, Saam B, Hagspiel KD, Johnson GA, Altes TA, de Lange EE, Kauczor HU. MRI of the lungs using hyperpolarized noble gases. Magn Reson Med 47: 1029-1051, 2002.
29. Morbach AE, Gast KK, Schmiedeskamp J, Dahmen A, Herweling A, Heussel CP, Kauczor HU, Schreiber WG. Diffusion-weighted MRI of the lung with hyperpolarized helium-3: a study of reproducibility. J Magn Reson Imaging 21: 765-774, 2005.

30. Mouded M, Egea EE, Brown MJ, Hanlon SM, Houghton AM, Tsai LW, Ingenito EP, Shapiro SD. Epithelial cell apoptosis causes acute lung injury masquerading as emphysema. Am J Respir Cell Mol Biol 41: 407-414, 2009.

31. O'Halloran RL, Holmes JH, Altes TA, Salerno M, Fain SB. The effects of SNR on ADC measurements in diffusion-weighted hyperpolarized He-3 MRI. J Magn Reson 185: 42-49, 2007.

32. Parraga G, Mathew L, Etemad-Rezai R, McCormack DG, Santyr GE. Hyperpolarized $3 \mathrm{He}$ magnetic resonance imaging of ventilation defects in healthy elderly volunteers: initial findings at 3.0 Tesla. Acad Radiol 15: 776-785, 2008.

33. Parraga G, Mathew L, Etemad-Rezai R, McCormack DG, Santyr GE. Hyperpolarized $3 \mathrm{He}$ magnetic resonance imaging of ventilation defects in healthy elderly volunteers: initial findings at 3.0 Tesla. Acad Radiol 15: 776-785, 2008.

34. Parraga G, Ouriadov A, Evans A, McKay S, Lam WW, Fenster A, Etemad-Rezai R, McCormack D, Santyr G. Hyperpolarized 3He ventilation defects and apparent diffusion coefficients in chronic obstructive pulmonary disease: preliminary results at 3.0 Tesla. Invest Radiol 42: 384-391, 2007.

35. Rabe KF, Hurd S, Anzueto A, Barnes PJ, Buist SA, Calverley P, Fukuchi Y, Jenkins C, Rodriguez-Roisin R, van Weel C, Zielinski J. Global strategy for the diagnosis, management, and prevention of chronic obstructive pulmonary disease: GOLD executive summary. Am J Respir Crit Care Med 176: 532-555, 2007.

36. Saam BT, Yablonskiy DA, Kodibagkar VD, Leawoods JC, Gierada DS, Cooper JD, Lefrak SS, Conradi MS. MR imaging of diffusion of (3)He gas in healthy and diseased lungs. Magn Reson Med 44: 174-179, 2000.

37. Salerno M, Altes TA, Mugler JP III, Nakatsu M, Hatabu H, de Lange EE. Hyperpolarized noble gas MR imaging of the lung: potential clinical applications. Eur J Radiol 40: 33-44, 2001.

38. Stamenovic D. Micromechanical foundations of pulmonary elasticity. Physiol Rev 70: 1117-1134, 1990.

39. Sukstanskii AL, Yablonskiy DA. In vivo lung morphometry with hyperpolarized 3He diffusion MRI: theoretical background. J Magn Reson 190: 200-210, 2008.

40. Swift AJ, Wild JM, Fichele S, Woodhouse N, Fleming S, Waterhouse J, Lawson RA, Paley MN, Van Beek EJ. Emphysematous changes and normal variation in smokers and COPD patients using diffusion 3He MRI. Eur J Radiol 54: 352-358, 2005.

41. Tanoli TS, Woods JC, Conradi MS, Bae KT, Gierada DS, Hogg JC, Cooper JD, Yablonskiy DA. In vivo lung morphometry with hyperpolarized $3 \mathrm{He}$ diffusion MRI in canines with induced emphysema: disease progression and comparison with computed tomography. J Appl Physiol 102: 477-484, 2007.

42. Weibel ER, Gill J. Structure-function relationships of the alveolar duct. In: Bioengineering Aspects of the Lung, edited by West JB. New York: Dekker, 1977, p. 730-738.

43. Wilson TA. Mechanics of the pressure-volume curve of the lung. Ann Biomed Eng 9: 439-449, 1981.

44. Wilson TA. Relations among recoil pressure, surface area, and surface tension in the lung. J Appl Physiol 50: 921-930, 1981.

45. Woodhouse N, Wild JM, Mills GH, Fleming S, Fichele S, Van Beek EJ. Comparison of hyperpolarized $3-\mathrm{He}$ administration methods in healthy and diseased subjects. In: Proceedings of the 14th Annual Scientific Meeting of the International Society of Magnetic Resonance in Medicine. Seattle, WA: International Society of Magnetic Resonance in Medicine, 2006, p. 1288.

46. Woodhouse N, Wild JM, Paley MN, Fichele S, Said Z, Swift AJ, Van Beek EJ. Combined helium-3/proton magnetic resonance imaging measurement of ventilated lung volumes in smokers compared with neversmokers. J Magn Reson Imaging 21: 365-369, 2005.

47. Woods JC, Choong CK, Yablonskiy DA, Bentley J, Wong J, Pierce JA, Cooper JD, Macklem PT, Conradi MS, Hogg JC. Hyperpolarized 3He diffusion MRI and histology in pulmonary emphysema. Magn Reson Med 56: 1293-1300, 2006.

48. Yablonskiy DA, Sukstanskii AL, Leawoods JC, Gierada DS, Bretthorst GL, Lefrak SS, Cooper JD, Conradi MS. Quantitative in vivo assessment of lung microstructure at the alveolar level with hyperpolarized 3He diffusion MRI. Proc Natl Acad Sci U S A 99: 3111-3116, 2002. 